\title{
VESTIBULAR AND ACOUSTIC DYSFUNCTION IN PATIENTS WITH EARLY STAGES OF VESTIBULAR SCHWANNOMA
}

\author{
Dmytro Zabolotny, Oleg Borysenko, Nina Mishchanchuk, Tetiana Shevtsova, Marin \\ Buracovschi \\ O. S. Kolomyichenko Institute of Otolaryngology, \\ National Academy of Medical Sciences of Ukraine, Kyiv, Ukraine
}

\begin{abstract}
INTRODUCTION: Vestibular schwannoma (VS) is known to be a benign tumor. It develops from the Schwann cells in the vestibular portion of the VIII nerve. The causes of tumor development are not fully defined. VS is diagnosed more often in people of active age (30-60 years), mostly in women.

AIM: The aim is to study the state of vestibular and acoustic dysfunctions in patients with VS at an early stage.

MATERIALS AND METHODS: A total of 71 patients with MRI-verified VS at an early stage were analyzed. The 1st group consisted of 34 patients with intracanal VS, and the 2 nd group included 37 patients with stage one VS.

Vestibular dysfunction, including the registration of three pathological vestibular reactions vestibulo-somatic, vestibulo-sensory and vestibulo-vegetative, was studied. Acoustic dysfunction was studied using tonal, speech and suprathreshold speech audiometry, and auditory brainstem responses (ABR).

RESULTS: Unilateral or asymmetric bilateral vestibular dysfunctions have been established in all patients at the early stages of VS, which forms phantom phenomena of sensory and motor memory in the cortical structures of the brain in the form of pathological vestibulo-somatic, vestibulo-sensory and vestibulo-vegetative reactions, which are to be considered during rehabilitation.

Acoustic dysfunction of different manifestations-from normal symmetrical hearing to socially adequate unilateral or asymmetric sensorineural hearing loss of degree I-II, and significant socially inadequate sensorineural hearing loss of degree III-IV, deafness, and also mixed hearing loss, was determined in these patients. ABR registration indicated unilateral or asymmetric delays of sound signals transferring in the brainstem structures at the early stages of VS with both normal hearing and socially adequately impaired hearing.
\end{abstract}

CONCLUSION: Various manifestations of vestibular and acoustic dysfunctions with development of excitatory and inhibitory processes in the peripheral, brainstem, and cortical structures of the vestibular and

Address for correspondence:

Oleg Borysenko

O. S. Kolomyichenko Institute of Otolaryngology

National Academy of Medical Sciences of Ukraine

3 Zoolohichna St

03057 Kyiv

Ukraine

e-mail:oleg_borysenko@ukr.net acoustic systems were defined in all patients at the early stages of VS.

Keywords: vestibular and acoustic dysfunctions, postural balance, spontaneous nystagmus, experimental nystagmus reaction, tonal and speech audiometry, $A B R$

Received: May 18, 2021

Accepted: June 24, 2021 


\section{INTRODUCTION}

Vestibular schwannoma (VS) is known to be a benign tumor. It develops from the Schwann cells in the vestibular portion of the VIII nerve (1-6). The causes of tumor development are not fully defined. VS is diagnosed more often in people of active age (30-60 years), mostly in women.

Despite the latest technologies, the disease duration-from the moment of initial symptom to the diagnosis, was as follows: less than one year was registered in $33 \%$ of patients, from 1 to 3 years-in $56.9 \%$, more than 3 years-in $18.8 \%$, according to the researchers from O.S. Kolomiychenko Institute of Otolaryngology, National Academy of Medical Sciences of Ukraine (7).

The vestibular system plays a decisive role in the spatial orientation at rest and in motion and in the formation of motor and sensory memory, which underlie all forms of sensory and motor activities. It responds to the development of disorders in the body, in particular, somatic, motor, sensory and autonomic ones with exceptionally subtle sensitivity, dynamism, objectivity of symptoms before other sensory systems, so it is of prime importance in regard to these functions (8-13).

According to the R. Jackler classification (14), VS at the early stages is an intracanally located in the internal auditory canal (IAC), and at stage one (I) leaves the IAC for the cerebellopontine angle at a distance of up to $10 \mathrm{~mm}$. Therefore, an important task of otoneurology is the detection of VS at the early stages, which are also called otiatric ones.

The unilateral or asymmetric manifestations of vestibular (VeD) and acoustic (AcD) dysfunctions are considered as a manifestation of VS, requiring the comprehensive clinical and electrophysiological examinations of the vestibular and auditory systems $(5,6,15,16)$.

If VS is suspected after these examinations, it is mandatory to perform MRI with contrast as the gold standard of objective diagnosis. It was noted that small VS may not grow for a long time, or grow slowly, not exceeding $1 \mathrm{~mm}$ per year (17). However, they can grow faster, filling the cerebellopontine angle, unilaterally disrupting the functions of the cranial nerves V, VI, VII. Progressing in growth, the VS also causes the functional disorders of brainstem struc- tures, which may become life-threatening. Detection of $\mathrm{VeD}$ and $\mathrm{AcD}$ in patients at the early clinical stages of VS is important for the provision of optimal medical care with minimal postoperative complications and effective and corrective rehabilitation.

\section{AIM}

The aim of the study was to evaluate the vestibular and acoustic dysfunctions in patients with VS at an early stage.

\section{MATERIALS AND METHODS}

A total of 71 patients with MRI-verified VS at an early stage were examined in the period 2015-2020 in the Department of Ear Microsurgery and Otoneurosurgery at the Kolomyichenko Otolaryngology Institute. Thirty of the patients were male and $41-\mathrm{fe}-$ male. The 1st group consisted of 34 patients with intracanal VS, and the 2 nd group included 37 patients with stage one VS.

Ten healthy individuals with normal vestibulometry and audiometry tests were chosen for the control group.

Vestibulometry was performed using the V.G. Bazarov scheme (8) with the assessment of postural balance (static balance by cephalography, kinetic balance and spatial orientation when performing Fukuda coordination tests such as writing and stepping ones).

Spontaneous, positional and experimental nystagmus responses induced by functional stimulations (caloric and rotational) were recorded by a computer electronystagmography (ENG) method with the neurocomplex of an SPE DX-system (Ukraine).

The nature of VeD was assessed by the I.B. Soldatov et al. method (18), and the degree of its manifestation-according to the V.G. Bazarov method (8) with the obligatory determination of the duration of vestibulo-sensory reactions, and the degree of manifestation of vestibulo-vegetative reflexes was assessed according to the K.L. Khilov method (19).

Auditory function was evaluated by MA31 (Germany) and Uteri (Denmark) audiometers. Thresholds of tonal and speech audiometry, legibility of speech tests, signs of paradoxical increase in their intelligibility at the maximum audiometer intensities from 110 to $120 \mathrm{~dB}$ were fixed, and ototopic disorders were also revealed by the Weber test. The degree of 
hearing loss was determined according to the international classification approved by the WHO.

Speech audiometry was performed under modern acoustic conditions (20).

Registration of $A B R$ was performed using the Eclipse analyzer system from Interacoustics in response to a click duration of $100 \mu$ s with a repetition rate of 21 per $1 \mathrm{sec}$ with an intensity of $80 \mathrm{~dB}$ above the subjective level of sound perception. A total of 1024 averaged evoked curves were analyzed using low- $(200 \mathrm{~Hz})$ and high-frequency $(2000 \mathrm{~Hz})$ filters with an epoch analysis within time intervals with 10 ms width.

The method of variation statistics and the Student's coefficient were used for the calculation of the significant differences between the values.

\section{RESULTS}

A retrospective analysis of complaints and anamnestic data of 71 individuals divided into two groups of patients with VS at an early stage was performed.

The first symptoms of $\mathrm{VeD}$ discomfort revealed various unilateral or asymmetric manifestations of three types of pathological reactions:

1. vestibulo-somatic spontaneous disorders of postural balance (functions of static and kinetic balance, disorders of spatial orientation without visual control);

2. vestibulo-sensory reactions (dizziness with an objective manifestation consisting of spontaneous or positional nystagmus of different intensity and direction);

3. vestibulo-vegetative reactions (nausea, vomiting, increased sweating, arrhythmias, changes in blood pressure).

Forms of motion sickness occurring for the first time or an exacerbation of such forms observed in childhood or adolescence were determined.

It is noteworthy that complaints of irritative processes in $\mathrm{VeD}$ were more often noted by persons from the 1st group. In patients from the 2 nd group the main complaints were disorders of balance and spatial orientation, especially in the dark, without visual control.

Spontaneous vestibulo-somatic disorders were detected in all 71 patients, namely: postural balance disorders (static and kinetic balance in 100\% of cases) and partially spontaneous or positional nystagmus.

At functional stimulation (caloric and rotational) the phenomena of unilateral or asymmetric hyperreflexia of experimental nystagmus reaction in $69 \%$ of the patients from the 1st group and dissociation-in $10 \%$ of cases registered by computer ENG.

Phenomena of unilateral or asymmetric bilateral hyporeflexia of the experimental nystagmus reactions were registered in $83 \%$ of the cases, and signs of dissociation-in $15 \%$ of the cases in the 2 nd group.

Pathological vestibulo-vegetative reactions were recorded in $65 \%$ of the cases from the 1st group, and in $52 \%$ of those from the 2nd group. Pathological vestibulo-sensory reactions were detected in $81 \%$ of the cases in group 1, and in 27\% of those in group 2 ( 3 times less than in the $1^{\text {st }}$ group).

Analyzing the vestibulometric examinations with functional stimulations, while taking into account three types of pathological vestibular reactions, first- or second-degree vestibular syndrome, with and without preservation of the vectorial rules, was established in VS patients with unilateral or asymmetric phenomena of hyperreflexia of the experimental nystagmus reaction from the 1st group, as well as in patients with unilateral or asymmetric phenomena of hyporeflexia of experimental nystagmus reaction from the 2 nd group.

Craniocerebral injuries, concomitant endocrine (diabetes mellitus, thyroid pathology), cardiovascular and digestive diseases, emergence motion disease or its aggravation, which were established in childhood or later, were present in the anamnesis of $54 \%$ patients from both groups.

"Phantom" (sensory) sensations are formed in the cortical structures of the brain in the form of dizziness, postural balance disorders, spatial orientation disorders, nausea, general weakness in vestibular dysfunction at the early stages of VS, as was noted by control examinations. The obtained data are consistent with those of other authors, who indicate the need to take into account the "phantom" sensations during rehabilitation $(21,22)$.

The dependence of the effect of rehabilitation on many factors was observed despite the high compensatory mechanisms in the vestibular cortex and the participation of other sensory organs. 
In particular, this dependence is clearly seen in different age categories (in younger age the compensation occurs faster, in older age lasts longer).

Identified pathological vestibular reactions (vestibulo-somatic, namely spontaneous and caused by functional stimulations, vestibulo-vegetative, and vestibulo-sensory) of vestibular dysfunction in patients with VS are presented on Fig. 1.

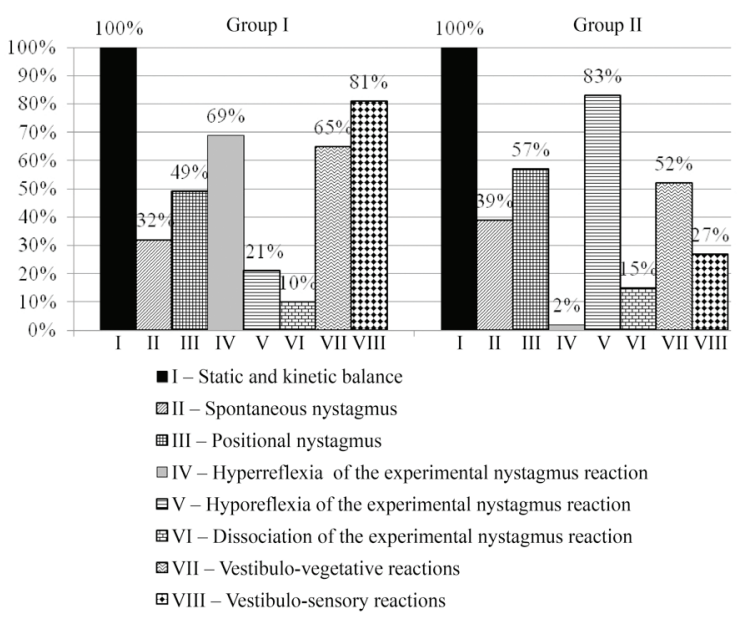

Fig. 1. Manifestations of pathological spontaneous reactions caused by vestibulo-somatic, vestibulovegetative and vestibulo-sensory reactions in vestibular dysfunction of VS patients.

Auditory discomfort was manifested by complaints of unilateral tinnitus, first periodic and then constant ones of varying tone and intensity in $92 \%$ of the patients from both groups.

Different hearing levels were noted in patients of both groups: normal symmetrical hearing, unilateral or asymmetric bilateral sensorineural hearing loss with slowing perception of speech tests, signs of paradoxical decrease of speech intelligibility with increased audiometer volume, complete deafness with ototopic disorders, and mixed hearing loss. The re- sults of the audiometric examinations are presented in Table 1.

According to audiometric examinations, normal bilateral symmetrical hearing was observed in 10 (29.4\%) patients from the first group, first- and second-degree unilateral sensorineural hearing loss-in 13 (38.2\%), first- and second-degree asymmetric bilateral sensorineural hearing loss-in 4 (12.8\%), second-degree mixed hearing loss-in 1 (2.9\%), belonging to socially adequate hearing impairments.

They achieved $100.0 \%$ intelligibility of speech tests, without signs of its slowing and a paradoxical decrease in intelligibility of speech tests with an audiometer intensity of 110-120 dB.

Third- and fourth-degree of socially inadequate hearing, hearing loss, was only observed in 4 patients (12.8\%) from this group. The signs of delayed comprehension of speech testing were revealed in these patients, which indicated the development of inhibitory processes in the auditory cortex. Along with this, the signs of a paradoxical decrease in speech intelligibility with increasing the volume of the audiometer to $110-120 \mathrm{~dB}$ were noted.

At the same time, they indicate disorders of excitation processes in the cortical structures of the vestibular system, which narrows the range of comfortable perception of speech tests, complicating social communication and creating obstacles to hearing correction by hearing aids.

Among 37 patients from the 2nd group, normal hearing was only registered in 2 (5.4\%), a first- and second-degree sensorineural hearing loss without disturbances of speech tests-in 16 (43.0\%). However, third- and fourth-degree sensorineural hearing loss was recorded in 14 patients (37.6\%), which was 3 times more frequent than in patients from the 1st group. Second-degree unilateral mixed hearing loss was registered in one patient from the 2 nd group.

Table 1. Acoustic dysfunction according to audiometry in VS patients.

\begin{tabular}{|c|c|c|c|c|c|c|c|c|c|c|c|}
\hline \multirow{3}{*}{ Groups } & \multirow{3}{*}{ Normalhearing } & \multicolumn{8}{|c|}{ Degrees of Sensorineural Hearing Loss } & \multirow{3}{*}{$\begin{array}{l}\text { Unilateral } \\
\text { Deafness }\end{array}$} & \multirow{3}{*}{$\begin{array}{c}\text { Unilateral } \\
\text { Mixed } \\
\text { Hearing Loss }\end{array}$} \\
\hline & & \multicolumn{4}{|c|}{ Unilateral } & \multicolumn{4}{|c|}{ Asymmetric } & & \\
\hline & & I & II & III & IV & I & II & III & IV & & \\
\hline 1 & 10 & 6 & 7 & 1 & 1 & 1 & 3 & 2 & - & 2 & 1 \\
\hline 2 & 2 & 3 & 12 & 4 & 3 & - & 1 & 4 & 3 & 4 & 1 \\
\hline Total & 12 & 9 & 19 & 5 & 4 & 1 & 4 & 6 & 3 & 6 & 2 \\
\hline
\end{tabular}


In addition, 6 out of 71 patients (8.5\%) with total unilateral sensorineural hearing loss (2 persons from the 1st group, and 4 persons from the 2nd group), with monoaural perception of hearing and significant ototopic disorders were analyzed. In such cases, patients could not determine which side received the sound or speech signal, creating dangerous situations, reducing proficiency and efficiency.

The results of interpeak latency (IPL) parameters of ABR in patients with normal hearing from both groups, first- and second-degree sensorineural hearing loss, compared to the persons of the control group are presented in Table 2 . mild symptoms. The natural history of the tumor supports abstaining from any treatment, shifting to an active policy if it grows or new, invalidating symptoms develop $(5,22)$. Active therapies such as microsurgery or radiotherapy may be offered at the time of diagnosis, but only if they provide the patient more benefit than observation alone $(12,19)$. It is essential to ask why and when a small VS should be actively treated. The goal of treatment should be to ensure long-term cure of the disease with no further morbidity on cranial nerves VII and VIII, or post-treatment sequelae.

Clinically, most VS patients present with uni-

Table 2. Average of interpeak latencies of ABR in VS patients and control group.

\begin{tabular}{|c|c|c|c|c|}
\hline \multirow{2}{*}{ Group } & \multirow{2}{*}{ Hearing Condition } & \multicolumn{3}{|c|}{ Interpeak Intervals (ms) of $\mathrm{ABR}(\mathrm{M} \pm \mathrm{m})$} \\
\hline & & I-III & III-V & $\mathrm{I}-\mathrm{V}$ \\
\hline Control & Norm & $2.15 \pm 0.02$ & $1.87 \pm 0.01$ & $4.02 \pm 0.02$ \\
\hline \multirow{3}{*}{ Group 1} & Norm & $2.20 \pm 0.02^{\star}$ & $2.12 \pm 0.03^{*}$ & $4.32 \pm 0.03^{*}$ \\
\hline & Degree I & $2.23 \pm 0.02$ & $2.21 \pm 0.03$ & $4.43 \pm 0.02$ \\
\hline & Degree II & $2.25 \pm 0.01$ & $2.26 \pm 0.02$ & $4.51 \pm 0.03$ \\
\hline \multirow{3}{*}{ Group 2} & Norm & $2.23 \pm 0.02 *$ & $2.12 \pm 0.05^{\star}$ & $4.35 \pm 0.04^{*}$ \\
\hline & Degree I & $2.26 \pm 0.02^{* *}$ & $2.21 \pm 0.05^{\star *}$ & $4.47 \pm 0.02^{\star *}$ \\
\hline & Degree II & $2.30 \pm 0.03^{* *}$ & $2.26 \pm 0.02^{* *}$ & $4.56 \pm 0.02^{* *}$ \\
\hline
\end{tabular}

Notes. ${ }^{*}$ - IPL of VS patients in the 2 groups with normal hearing were significantly prolonged in comparison with the control group, $p<0.05$;

** - IPL were significantly prolonged in patients from the 2nd group in comparison with the 1st group, $p<0.05$.

It should be noted that, although according to audiometric examinations normal hearing was recorded symmetrically on both sides in 10 patients of the 1st group and in 2 patients of the 2 nd group, significant asymmetric changes in IPL of ABR were registered.

They were detected in the 2 groups of patients as an increase in the IPL parameters I-III, III-V and their total value I-V on the side of VS compared with the control group, as well as with the contralateral ear.

The obtained results indicate the changes in the brainstem structures of the auditory system in patients with VS and socially adequate hearing, as well as in those with a normal hearing.

\section{DISCUSSION}

Thanks to early imaging, VS are now diagnosed more frequently and are often small, presenting with lateral sensorineural hearing loss (94\%) and tinnitus $(83 \%)$. The frequency of the vestibular symptoms like vertigo and unsteadiness varies widely (17-75\% of patients), but they are likely underreported (21). In the early stages, VS may not manifest itself clinically. Sometimes a tumor is discovered by accident during an MRI scan of the brain. Diagnosis of a tumor in the early stages remains a challenging task for otoneurology.

In the early stages of VS, when the tumor is just starting to grow on the vestibular nerve, nerve irritation is observed. Patients may complain of slight dizziness, and vestibulometry can reveal hyperreflexia from the vestibular analyzer.

A total of $69 \%$ of patients with intracanal VS had hyperreflexia of the nystagmus reaction in our observations. As the tumor grows, compensation for the disorders of the vestibular system and loss of 
function of the vestibular nerve destroyed by the tumor occurs. In VS patients with a $1^{\text {st }}$ stage tumor, we have already observed hyporeflexia of experimental nystagmus in $83 \%$ of the cases.

Patients with VS may have unilateral hearing impairment of varying severity, or may not have hearing impairment at all. The degree of hearing impairment does not indicate the size of the tumor or its presence. The registration of auditory potentials, even with a normal hearing level, makes it possible to suspect a tumor. At the same time, an increase in IPL between peaks I-III and I-V on the side of the tumor is recorded even with small intracanal tumors with a size of 2-3 mm. In our observations, we noted an increase in the mean values of IPL in both groups, even with a normal hearing level on the side of the tumor.

It should be emphasized that the disturbances of inhibitory and excitatory processes in the auditory cortex registered in the speech discrimination score in VS patients with socially inadequate hearing from both groups complicate social communication significantly.

When comparing the results of the examinations of patients with VS with significant binaural hearing loss with a difference of more than $30 \mathrm{~dB}$, and with third- and fourth-degree unilateral hearing loss or unilateral deafness with asymmetry of experimental nystagmus reactions, significant equilibrium disturbances were registered in comparison with less important changes in vestibulometry parameters in first- and second-degree unilateral hearing loss.

According to our data, taking into account the ototopic disorders in AcD together with the changes in postural balance in $\mathrm{VeD}$ is the important point in a comprehensive objective assessment of disorders of spatial orientation in the expert determination of proficiency and efficiency.

\section{CONCLUSION}

1. Vestibular dysfunction in all patients at the early stages of VS is manifested by unilateral or asymmetric disturbance of postural balance, unilateral or asymmetric disorders of experimental nystagmus responses during the functional stimulation: first- and second-degree processes of excitation or inhibition with or without preservation of vectorial rules.
2. Vestibular dysfunction in patients with VS forms the "phantom" phenomena of sensory, and motor memory such as dizziness, postural balance disorders, nausea and other autonomic disorders in the cortical structures of the brain. These facts are of great importance for the rehabilitation measures.

3. Acoustic dysfunction in patients at the early stages of VS can be different from symmetrical normal hearing to first- and second-degree socially adequate unilateral or asymmetric bilateral sensorineural hearing loss and third- and fourth-degree unilateral or bilateral socially inadequate hearing loss, also deafness as well as mixed hearing loss.

4. Unilateral or asymmetric prolongations in interpeak latencies of $\mathrm{ABR}$ were revealed. They indicate unilateral or asymmetric preclinical delay of sound signals transferring in patients at the early stages of VS in the brain auditory system, which is important for differential diagnosis.

5. In patients with socially inadequate hearing from both groups, the disorders of inhibitory and excitatory processes in the auditory cortex were registered during speech audiometric testing, which significantly complicated their social communication.

Thus, the comprehensive diagnostic approach to the detection of vestibular and acoustic dysfunctions in patients at the early stages of VS is necessary for optimal choice of treatment, preventive and rehabilitative corrective measures to more quickly and effectively achieve compensation of impaired functions to maintain proficiency, efficiency and socialization in an individual's life.

\section{Conflict of Interest}

There is no conflict of interest.

\section{Acknowledgements}

This article is dedicated to the memory of the Teacher, Professor Atanas Kehajov.

\section{REFERENCES}

1. Atanas Kehajov. Differential-diagnostic problems in otoneurology. Sofia: Medicine and Physical Education; 1972. 
Dmytro Zabolotny, Oleg Borysenko, Nina Mishchanchuk et al.

2. Chen M, Fan Z, Zheng X, Cao F, Wang L. Risk factors of acoustic neuroma: Systematic review and meta-analysis. Yonsei Med J. 2016; 57(3):776-83. doi: 10.3349/ymj.2016.57.3.776-783.

3. Dunn IF, Bi WL, Mukundan S, Delman BN, Parish J, Atkins T, et al. Congress of Neurological Surgeons systematic review and evidence-based guidelines on the role of imaging in the diagnosis and management of patients with vestibular schwannomas. Neurosurgery. 2018;82(2):E32-4. doi: 10.1093/ neuros/nyx 510 .

4. Goldbrunner R, Weller M, Regis J, Lund-Johansen $\mathrm{M}$, Stavrinou P, Reuss D, et al. EANO guideline on the diagnosis and treatment of vestibular schwannoma. Neuro Oncol. 2020;22(1):31-45. doi:10.1093/ neuonc/noz153.

5. Stangerup SE, Tos M, Thomsen J, Caye-Thomasen P. Hearing outcomes of vestibular schwannoma patients managed with 'wait and scan': predictive value of hearing level at diagnosis. J Laryngol Otol. 2010; 124(5):490-4. doi: 10.1017/ S0022215109992611.

6. Hong CS, Moliterno J. The patient-centered approach: a review of the literature and its application for acoustic neuromas. J Neurol Surg B Skull Base. 2020;81(3):280-6. doi:10.1055/s-0039-16923961.

7. Zabolotnyy DI, Sushko YuA., Borisenko ON, Srebnyak IA, Golod AN, Zlyy MV. Early diagnosis of acoustic neurinoma. Rossiyskaya otorinolaringologiya. 2003;3:70-5. (in Russian).

8. Bazarov VG. Clinical vestibulometry. Kyiv: Zdorovia; 1988. (in Russian).

9. Pedachenko YeG, Skobskaya OYe, Gudkov VV, Malysheva AYu. Problems of diagnosis of vestibular schwannomas. Ukrayins'kyy neyrokhirurhichnyy zhurnal. 2014; 1:53-6. (in Russian).

10. Blödow A, Helbig R, Wichmann N, Wenzel A, Walther LE, Bloching MB. Video-Kopfimpulstest oder thermische Prüfung? Zeitgemäße Funktionsdiagnostik des Vestibularisschwannoms (Video head impulse test or caloric irrigation? Contemporary diagnostic tests for vestibular schwannoma). HNO. 2013;61(9):781-5. doi: 10.1007/s00106-013-2752-x. (in German).

11. Cao Z, Zhao F, Mulugeta H. Noise exposure as a risk factor for acoustic neuroma: a systematic review and meta-analysis. Int J Audiol. 2019;58(9):525-32. doi:10.1080/14992027.2019.1602 289.
12. Leon J, Lehrer EJ, Peterson J, Vallow L, Ruiz-Garcia $\mathrm{H}$, Hadley A, et al. Observation or stereotactic radiosurgery for newly diagnosed vestibular schwannomas: A systematic review and meta-analysis. J Radiosurg SBRT. 2019;6(2):91-100.

13. Zabolotnyy DI, Mishchanchuk NS. Vestibular system: Anatomy, physiology and clinical evaluation. In: Suzuki T, editor. Somatosensory and motor research. London, United Kingdom: IntechOpen; 2020. pp. 35-54.

14. Jackler R, Driscoll CLW. Tumor of the ear and temporal bone. Lippincot Williams \& Wilkins; 2000. p. 172-218.

15. Lin EP, Crane BT. The management and imaging of vestibular schwannomas. Am J Neuroradiology. 2017;38(11):2034-43. doi: 10.3174/ajnr.A5213.

16. Rosahl S, Bohr C, Lell M, Hamm K, Iro H. Diagnostics and therapy of vestibular schwannomas an interdisciplinary challenge. GMS Curr Top Otorhinolaryngol Head Neck Surg. 2017;16:Doc03. doi:10.3205/cto000142.

17. Kirchmann M, Karnov K, Hansen S, Dethloff T, Stangerup SE, Caye-Thomasen P. Ten-year followup on tumor growth and hearing in patients observed with an intracanalicular vestibular schwannoma. Neurosurgery. 2017;80(1):49-56. doi: 10.1227/ NEU.0000000000001414.

18. Soldatov IB, Sushcheva GP, Khrappo NS. Vestibular dysfunction. Moskva: Meditsina; 1980. (in Russian).

19. Khilov KL. The function of the organ of balance and illness of movement. Leningrad: Meditsina; 1969. (in Russian).

20. Mishchanchuk NS, Radchenko OI, Karamzina LA. Speech audiometry for clinical audiology in modern conditions. Zhurnal Ushnykh, Nosovykh i Horlovykh Khvorob. 2016;2:49-53. (in Ukrainian).

21. Andersen JF, Nilsen KS, Vassbotn FS, Møller P, Myrseth E, Lund-Johansen M, Goplen FK. Predictors of vertigo in patients with untreated vestibular schwannoma. Otol Neurotol. 2015;36(4):647-52. doi: 10.1097/MAO.0000000000000668.

22. Stangerup SE, Caye-Thomasen P, Tos M, Thomsen J. The natural history of vestibular schwannoma. Otol Neurotol 2006; 27(4):547-52. doi: 10.1097/01mao0000217356.73463.e7. 